\title{
Quantitative Comparison of Five Different Photosensitizers for Use in a Photopolymer
}

\author{
Yue Qi, ${ }^{1}$ Michael R. Gleeson, ${ }^{2}$ Jinxin Guo, ${ }^{1}$ Sergi Gallego, ${ }^{3}$ and John T. Sheridan ${ }^{1}$ \\ ${ }^{1}$ School of Electrical, Electronic, and Communications Engineering, Communications and Optoelectronic Research Centre, \\ The SFI-Strategic Research Cluster in Solar Energy Conversion, College of Engineering and Architecture, University College Dublin, \\ Belfield, Dublin 4, Ireland \\ ${ }^{2}$ Department of Computer Science, NUIM, Maynooth, Kildare, Ireland \\ ${ }^{3}$ Departamento de Física, Ingeniería de Sistemas y Teoría de la Señal, Universidad de Alicante, Apartado 99, \\ 03080 Alicante, Spain
}

Correspondence should be addressed to John T. Sheridan, john.sheridan@ucd.ie

Received 2 May 2012; Accepted 19 July 2012

Academic Editor: Yasuo Tomita

Copyright () 2012 Yue Qi et al. This is an open access article distributed under the Creative Commons Attribution License, which permits unrestricted use, distribution, and reproduction in any medium, provided the original work is properly cited.

\begin{abstract}
Several studies of the time varying photon absorption effects, which occur during the photoinitiation process involving in photopolymer materials, have been presented. Three primary mechanisms have been identified: (i) the dye absorption, (ii) recovery, and (iii) bleaching. Based on an analysis of these mechanisms, the production of primary radicals can be physically described and modelled. In free radical photopolymerization systems, the excited dye molecules induce the production of the primary radicals, $R^{\bullet}$, which are key in determining how many monomers are polymerized. This, in turn, is closely related to the refractive index modulation formed during holographic recording. In this paper, to avoid the complexities involved in estimating the rate constant of intersystem crossing, $k_{s t}$, in going from the excited singlet state dye to the excited triplet state dye, we introduce two rates, $k_{a S}$ and $k_{a T}$ these are the proposed rate constants of photon absorption in going from the ground state to the singlet and triplet states, respectively. Using the resulting model, four kinds of Xanthene dyes: Erythrosin B; Eosin Y; Phloxine B, Rose Bengal, and one Thiazine dye: Methylene Blue, are experimentally characterised for use in an AA/PVA photopolymer.
\end{abstract}

\section{Introduction}

Photopolymer materials and the photochemical kinetics associated with them [1-3] are being actively studied for practical applications [4-8] such as 3D hybrid optoelectronic circuits, holographic storage [9-13], photoembossing (including the manufacturing of refractive and diffractive optical elements), metrology, 3D displays, and the selftrapping of light [14].

During the photopolymerisation process, the initial step, which involves the absorption of light by a dye, eventually results in the generation of radicals, and this initiation process plays a critical role in grating formation. The theoretical model of the photochemical processes has been developed to include the effects of photosensitizer recovery and bleaching [15-18], in order to permit the accurate prediction of the resulting material behaviour.
In a previous model of this material, in order to simplify the modelling of intersystem crossing from the excited singlet state dye to the excited triplet state dye, a bulk parameter $\Psi$ was introduced [18]. It was assumed that during exposure a constant fraction $\Psi$ of the ground state molecules present was always available to be converted into the singlet state, while $(1-\Psi)$ of them are available to be converted into the triplet excited state. In [19], a more physical representation of intersystem crossing, occurring at a rate constant, $k_{s t}$, was introduced, but this rate is difficult to estimate by the method described in [19] in that the monomer will be quickly crystallised when triethanolamine is not included in the layer. In order to overcome the limitations of these models, in this paper, we simplify our approach by introducing two new rates, $k_{a s}$ and $k_{a T}$, into the model, which are the rate constant of photon absorption from ground state to singlet state and triplet state, respectively. Then using this simplified 
model, the behaviours of five different photosensitizers, in an acrylamide polyvinyl alcohol (AA/PVA) photopolymer material, are studied. Detailed experimental and theoretical analyses are presented.

The paper is organized as follows. In Section 2, we introduce the photochemical reactions that take place and the NPDD model, including descriptions of the processes of initiation, propagation, termination, and inhibition. In Section 3, the material preparation is presented. In Section 4, the photoabsorptive behaviours of four green-sensitive types photosensitizer: Erythrosine B (EB); Eosin Y (EY); Phloxine $\mathrm{B}(\mathrm{PB})$, Rose Bengal (RB), of the same molarity in equivalent PVA/AA layers, and one red-sensitive photosensitizer, Methylene Blue (MB), of a higher molarity (due to the weaker absorptivity at the wavelength of $633 \mathrm{~nm}$ ), also in a PVA/AA layer, are experimentally examined. Based on these results, the recovery and bleaching behaviours of these dyes are also estimated. In Section 5, physical parameter values extracted by fitting the measured results using our model are presented and analysed. Finally, in Section 6, a brief conclusion is given.

\section{Theoretical Analysis of Photopolymer Material}

In free radical photopolymerization systems, the excited dye molecules induce the production of the primary radicals, $R^{\bullet}$ the concentration of which is key in determining how much monomers are polymerized. This, in turn, is closely related to the refractive index modulation formed during holographic recording.

In this paper, nonlocal photopolymerization driven diffusion (NPDD) model is developed. This model includes the effects of (a) the kinetics of the major photochemical processes; (b) the temporally and spatially varying photon absorption; (c) the nonlocal material response.

2.1. Photochemical Reactions. In the case of free radical photopolymerization systems, the kinetic model describing what takes place involves four main processes [2, 16, 20-23], (I) initiation, (II) propagation, (III) termination, and (IV) Inhibition. We note that each may involve several physicochemical reactions. We highlight the major chemical reactions in each process $[3,20,24-27]$. A summary of the discussion in this section is given in the flow chart in Figure 1. The excitation of the Xanthenes dyes is similar and is due to the breaking up of the $\mathrm{C}=\mathrm{O}$ double bond when exposed to the green beam [28]. Since the structure of Methylene Blue is different from the Xanthene dye, the excitation is also different, that is, due to the breaking up of the $\mathrm{C}=\mathrm{N}$ bond when exposed to the red beam [29]. However the reactions between Methylene Blue and the electron donor are similar to the case of Xanthenes dyes. The detailed chemical scheme of the photopolymerisation is described in $[20,28,29]$. The reactions highlighted within the dashed box will be studied experimentally in Section 4. In particular the introduction of $k_{a S}$ and $k_{a T}$ should be noted when comparing this model to previous models.
(I) Initiation. During illumination, the reaction between the photosensitiser and the electron donor (coinitiator) leads to the production of initiator radicals, $R^{\bullet}$, which can react with the monomers to produce chain initiators, $M^{\bullet}$.

$$
\begin{gathered}
D+h \nu \stackrel{k_{a S}}{\longrightarrow}{ }^{1} D^{*} \\
D+h \nu \stackrel{k_{a T}}{\longrightarrow}{ }^{3} D^{*} \\
{ }^{1} D^{*} \stackrel{k_{r}}{\longrightarrow} D \\
{ }^{3} D^{*}+E D \stackrel{k_{d}}{\longrightarrow} R^{\bullet}+H^{+}+D^{\bullet-} \longrightarrow R^{\bullet}+H D^{\bullet} \\
E D+H D^{\bullet} \stackrel{k_{b}}{\longrightarrow} H_{2} D+E D_{\text {int }} \\
R^{\bullet}+M \stackrel{k_{i}}{\longrightarrow} M_{1}^{\bullet},
\end{gathered}
$$

where $D$ represents the photosensitizer molecule, $h v$ indicates the energy absorbed from a photon, $k_{a S}=\varphi_{S} \times \varepsilon \times d \times I_{0}^{\prime}$, $k_{a T}=\varphi_{T} \times \varepsilon \times d \times I_{0}^{\prime}\left(\varphi_{S}\right.$ and $\varphi_{T}$ are the quantum efficiencies of the reactions in which the ground state dye is converted into the singlet ${ }^{1} D^{*}$ and triplet ${ }^{3} D^{*}$ dye states, $\varepsilon$ is the dye molar absorptivity, $d$ is the thickness of the layer, and $I_{0}^{\prime}$ Einstein's $/ \mathrm{cm}^{3} \mathrm{~s}$ is the incident intensity [27]) are the rate constants of photon absorption in ground state, $k_{r}$ is the recovery rate of the dye from singlet excited state to ground state, including the processes of (1) radiationless energy transfer to another molecule such as triethanolamine which acts as an electron donor (ED), (2) the emission of a photon by fluorescence $[16,18]$. The recovery of triplet state dye is ignored for simplicity and also due to the even lower recovery rate in this process $[18,19], E D$ is the electron donor, and $k_{d}$ is the rate constant of electron donation through which $E D$ becomes a free radical, $R^{\bullet}, H D^{\bullet}$ represents a radicalized dye, which abstracted a hydrogen ion from the electron donor, $k_{b}$ is the rate constant of the photobleaching process, that is, the rate formation of dihydro dye, $H_{2}$ Dye, $E D_{\text {int }}$ is an intermediate form of the electron donor, $k_{i}$ is the chain initiation kinetic constant, and $M$ represents a monomer molecule. We note that (1a) and (1b) are new differing from the models proposed in $[18,19]$.

(II) Propagation. The chain initiator, $M_{1}^{*}$ will attach itself to another monomer molecule, $M$, by addition to the $\mathrm{C}=\mathrm{C}$ bond yielding a growing polymer radical with an active tip. Through propagation polymer chain growth then follows [20].

$$
M_{n}^{\bullet}+M \stackrel{k_{p}}{\longrightarrow} M_{n+1}^{\bullet}
$$

where $k_{p}$ is the rate constant of propagation and $M_{n}^{\bullet}$ and $M_{n+1}^{\bullet}$ are the growing macroradical chains of length $n$ and $(n+1)$ repeat monomeric units $(n \geq 1)$.

(III) Termination. Termination can occur in three ways. Two of these, disproportionation and combination, involve two 


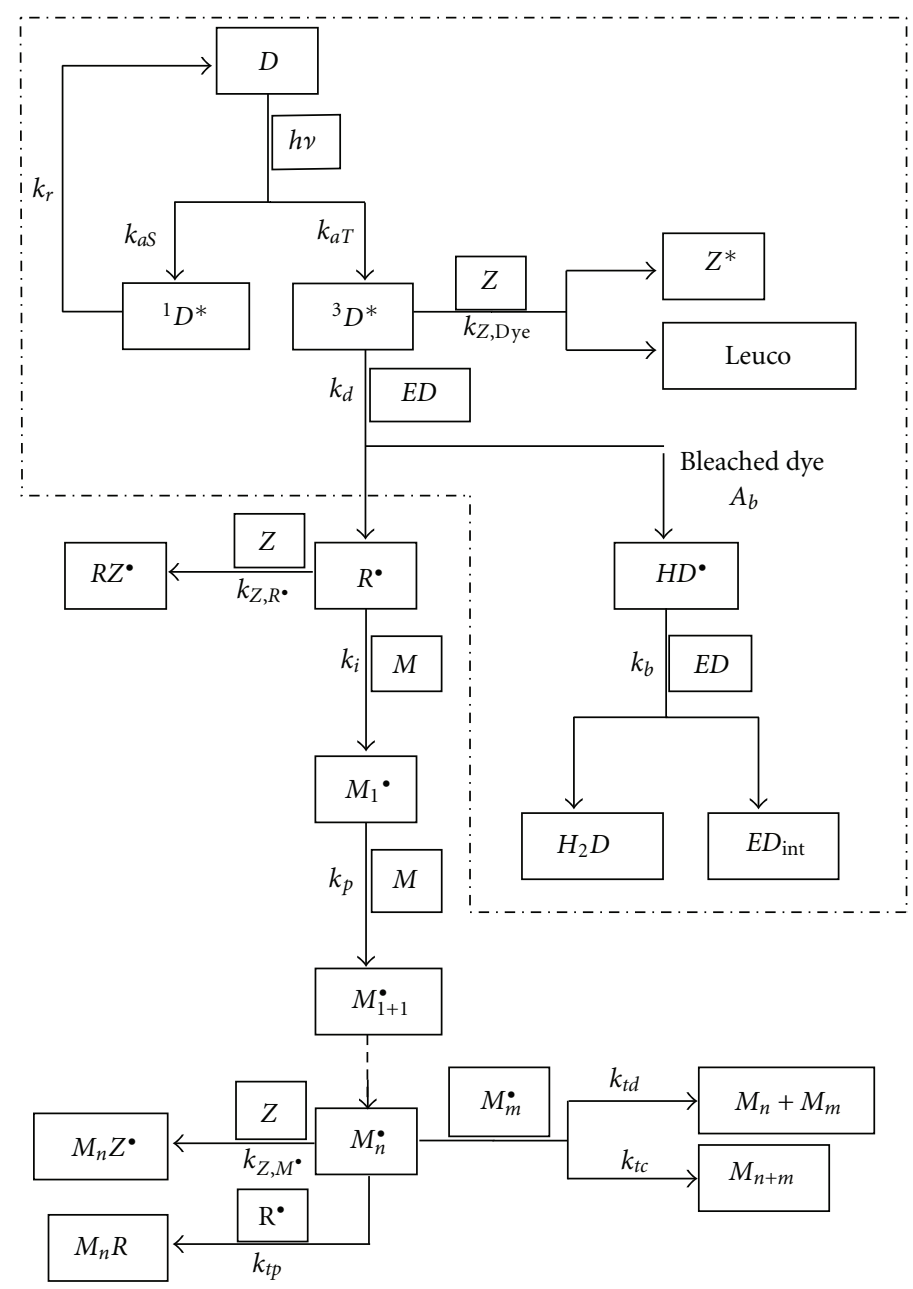

FIGURE 1: Flow chart of the photochemical process [16]. We note that the introduction of $k_{a S}$ and $k_{a T}$ is new. (All the parameters and variables are defined in the analysis in the text.)

growing macroradicals interacting, that is, the bimolecular termination mechanism.

$$
\begin{gathered}
M_{n}^{\bullet}+M_{m}^{\bullet} \stackrel{k_{t c}}{\longrightarrow} M_{n+m} \\
M_{n}^{\bullet}+M_{m}^{\bullet} \stackrel{k_{t d}}{\longrightarrow} M_{n}+M_{m},
\end{gathered}
$$

where $k_{t c}$ and $k_{t d}$ are the rate constants of combination and disproportionation termination respectively. $M_{n}^{\bullet}, M_{m}^{\bullet}$, and $M_{m+n}^{*}$ represent terminated chains which have no radical tip, that is, a dead polymer. In this analysis, $k_{t c}$ and $k_{t d}$ will be treated as a single lumped rate constant, $k_{t}=k_{t c}+k_{t d}$ $\left(\mathrm{cm}^{3} \mathrm{~mol}^{-1} \mathrm{~s}^{-1}\right)$, as the mode of termination does not effect the polymerization kinetics [25].

A third possible termination mechanism involves primary radical termination $[19,26]$.

$$
M_{n}^{\bullet}+R^{\bullet} \stackrel{k_{t p}}{\longrightarrow} M_{n} R,
$$

where $k_{t p}$ is the rate constant of primary radical termination. In this step, a growing macroradical chain reacts with a primary radical (initiator radical) leading once again to the production of inactive or dead polymer chains [25].
(IV) Inhibition. Inhibitors are chemicals which react with the initiating and propagating radical species by rapidly removing or scavenging these radicals. Polymerization is completely halted until they are all consumed [24]. Several possible inhibitor reaction mechanisms are listed in the following:

$$
\begin{array}{r}
R^{\bullet}+Z \underset{k_{z, R^{\bullet}}}{\longrightarrow}\left(R Z^{\bullet} \text {, and/or } R+Z^{\bullet}\right) \\
{ }^{3} D^{*}+Z \stackrel{k_{z, \text { Dye }}}{\longrightarrow} \text { leuco }- \text { dye }+Z^{*} \\
M_{n}^{\bullet}+Z \stackrel{k_{z, M^{*}}}{\longrightarrow}\left(M_{n} Z^{\bullet} \text {, and } / \text { or } M_{n}+Z^{\bullet}\right),
\end{array}
$$

where $Z$ is the inhibitor species, for example, oxygen, ${ }^{3} D^{*}$ is the excited photosensitiser, $Z^{\bullet}$ is the concentration of singlet oxygen $[21,24,27]$, and $k_{z, R^{\bullet}}, k_{z \text {,Dye* }}$ and $k_{z, M^{*}}$ are the rate constants of inhibition of the primary radicals [15-17, 21, 30 ], the photosensitiser and the macroradicals respectively. Inhibition leads to a dead band at the start of exposure, that is, stopping grating formation during the initial exposure. The effects of inhibitors are especially significant when lower exposure energies (dose) are used, for example, when large 
areas must be exposed (low intensities) or short duration exposures must be used [27].

2.2. Inclusion in the NPDD Model. Based on the analysis present above and summarised in Figure 1, a set of coupled differential equations representing the spatial and temporal evolutions of the material concentrations associated with (1a)-(5c) can be derived using the same notation and following the same methodology as in references [24-26].

$$
\begin{aligned}
& \frac{d D(x, t)}{d t}=k_{r}{ }^{1} D^{*}(x, t)-k_{a S} D(x, t)-k_{a T} D(x, t), \\
& \frac{d^{1} D^{*}(x, t)}{d t}=k_{a S} D(x, t)-k_{r}^{1} D^{*}(x, t), \\
& \frac{d^{3} D^{*}(x, t)}{d t}=k_{a T} D(x, t)-k_{d}^{3} D^{*}(x, t) E D(x, t) \\
& -k_{z, \mathrm{Dye}^{*}}{ }^{3} D^{*}(x, t) Z(x, t),
\end{aligned}
$$

$\frac{d E D(x, t)}{d t}=-k_{d}^{3} D^{*}(x, t) E D(x, t)-k_{b} E D(x, t) H D^{\bullet}(x, t)$,

$$
\begin{aligned}
\frac{d R^{\bullet}(x, t)}{d t}= & k_{d}{ }^{3} D^{*}(x, t) E D(x, t)-k_{i} R^{\bullet}(x, t) u(x, t) \\
& \quad-k_{t p} M^{\bullet}(x, t) R^{\bullet}(x, t)-k_{z, R^{\bullet}} R^{\bullet}(x, t) Z(x, t),
\end{aligned}
$$

$$
\frac{d H D^{\bullet}(x, t)}{d t}=k_{d}{ }^{3} D^{*}(x, t) E D(x, t)-k_{b} E D(x, t) H D^{\bullet}(x, t)
$$

$$
\begin{aligned}
& \frac{d u(x, t)}{d t}=\frac{d}{d x}\left[D_{m}(x, t) \frac{d u(x, t)}{d x}\right]-k_{i} R^{\bullet}(x, t) u(x, t) \\
& -\int_{-\infty}^{\infty} k_{p} M^{\bullet}\left(x^{\prime}, t\right) u\left(x^{\prime}, t\right) G\left(x, x^{\prime}\right) d x^{\prime}, \\
& \frac{d N(x, t)}{d t}=\int_{-\infty}^{\infty} k_{p} M^{\bullet}\left(x^{\prime}, t\right) u\left(x^{\prime}, t\right) G\left(x, x^{\prime}\right) d x^{\prime} \\
& -\frac{d}{d x}\left[D_{N}(x, t) \frac{d N(x, t)}{d x}\right] \\
& \frac{d M^{\bullet}(x, t)}{d t}=k_{i} R^{\bullet}(x, t) u(x, t)-k_{t}\left[M^{\bullet}(x, t)\right]^{2} \\
& -k_{t p} M^{\bullet}(x, t) R^{\bullet}(x, t)-k_{Z, M} \cdot M^{\bullet}(x, t) Z(x, t), \\
& \frac{d Z(x, t)}{d t}=\frac{d}{d x}\left[D_{Z} \frac{d Z(x \cdot t)}{d x}\right]-k_{Z, \text { Dye }^{*}}{ }^{3} D^{*}(x, t) Z(x, t) \\
& -k_{Z, R} \cdot R^{\bullet}(x, t) Z(x, t)-k_{Z, M} \cdot M^{\bullet}(x, t) Z(x, t),
\end{aligned}
$$

where $u(x, t), N(x, t)$, and $M^{\bullet}(x, t)$ are the concentrations of free-monomer, polymer, and macroradical. $D_{m}(x, t)$, $D_{N}(x, t)$, and $D_{z}$ are the diffusion rates of monomer, polymer, and inhibitor respectively. Equation (6) arises due to the new proposed reactions in (1a) and (1b). Furthermore,
TABLe 1: Composition of AA/PVA photopolymer material.

\begin{tabular}{lcl}
\hline Component & Function & Amount per $100 \mathrm{ml}$ \\
\hline Poly-vinylalcohol (PVA) & Binder & $70 \mathrm{~cm}^{3}$ of $10 \%$ sol. \\
Acrlyamide (AA) & Monomer & $2.4 \mathrm{~g}$ \\
Bis-acrylamide & Cross-linker & $0.8 \mathrm{~g}$ \\
& & $16 \mathrm{~cm}^{3}$ of $1.25 \times$ \\
Xanthene & & $10^{-4} \mathrm{M}$ for Xanthene \\
dyes/Methylene Blue & Dye & dyes and $16 \mathrm{~cm}^{3}$ of 2 \\
& & $\times 10^{-4} \mathrm{M}$ for \\
& & Methylene Blue \\
Triethanolamine (TEA) & Electron Donor & $8 \mathrm{~cm}^{3}$ \\
& $(E D)$ & \\
\hline
\end{tabular}

we note that a rate equation governing the total bleached photosensitizer concentration can be obtained:

$$
\frac{d A_{b}(x, t)}{d t}=k_{Z, \text { Dye }^{*}} D^{*}(x, t) Z(x, t)+k_{b} E D(x, t) H D^{\bullet}(x, t),
$$

where $A_{b}$ denotes the total (leuco and dihydro) bleached photosensitizer concentration [19]. (see Figure 1).

In (10), $G\left(x, x^{\prime}\right)$ is the nonlocal material spatial response function given by [24]:

$$
G\left(x, x^{\prime}\right)=\frac{1}{\sqrt{2 \pi \sigma}} \exp \left[\frac{-\left(x-x^{\prime}\right)^{2}}{2 \sigma}\right],
$$

in which $\sigma$ is the constant nonlocal response parameter $[20,31]$. The nonlocal spatial response function represents the effect of initiation at a location $x^{\prime}$ on the amount of monomer polymerized at location $x$ [31].

2.3. Summary. In this section, we have shown how the new dye model can be included in the NPDD model, in which the processes of initiation, propagation, termination and inhibition are included. A method to overcome the limitations of the use of the parameter $\Psi$ in [18] and the intersystem crossing rate constant in the previous model [19] is presented. We introduce $k_{a s}$ and $k_{a T}$ into the model equations (1a) and (1b), which are the rate constant of photon absorption from ground state to singlet state and triplet state respectively. This leads to the coupled differential equation (6). This new model simplifies the modelling and data fitting of the experimental data in Section 4.

\section{Material Preparation}

Photopolymer materials are made sensitive to a particular wavelength using photosensitizing dye. The photosensitisers examined in this paper are that is, Xanthene dyes Erythrosin B (EB), Eosin Y (EY), Phloxine B (PB) and Rose Bengal (RB). These allow holographic recording to be carried out using a $532 \mathrm{~nm}$ Solid-State Crystal Laser. We have also sensitized our AA/PVA material in the red ( $\mathrm{HeNe} 633 \mathrm{~nm}$ ) using Methylene Blue $(\mathrm{MB})$, which is a Thiazine dye.

Our PVA/AA material was made using the components listed in Table 1. The material was prepared as follows: 
(a) $10 \mathrm{~g}$ of PVA was added to $100 \mathrm{~cm}^{3}$ of deionised water and dissolved using a heater/stirrer. This solution is then allowed to cool and then $70 \mathrm{~cm}^{3}$ of this solution was transferred into a beaker.

(b) $8 \mathrm{~cm}^{3}$ of Triethanolamine (ED) was added to the PVA solution and stirred thoroughly.

(c) $2.4 \mathrm{~g}$ of Acrlyamide and $0.8 \mathrm{~g}$ of Bis-acrylamide were added to the PVA solution under a fume cupboard and stirred until completely dissolved.

(d) $16 \mathrm{~cm}^{3}$ of $1.25 \times 10^{-4} \mathrm{M}$ Xanthene dye (EB/EY/ $\mathrm{FB} / \mathrm{RB})$ or $16 \mathrm{~cm}^{3}$ of $2 \times 10^{-4} \mathrm{M}$ Methylene Blue $(\mathrm{MB})$ was then added to the beaker. As indicated in Section 1, a higher concentration of $\mathrm{MB}$ is used due to its weaker absorptivity at the wavelength of $633 \mathrm{~nm}$. This step and subsequent steps were carried out under a safety light, as the material is now sensitive to green/red light.

(e) The solution is then made up to $100 \mathrm{~cm}^{3}$ in a volumetric flask with de-ionised water.

(f) The solution is then stored in the dark ready for plate preparation.

To prepare dry material layers for holographic exposure the solution prepared above is used as follows:

(a) The microscope glass slice on which the material is to be deposited $(75 \mathrm{~mm} \times 25 \mathrm{~mm}$ ) is cleaned thoroughly using de-ionised water and Acetone. Once cleaned the plates are placed on a level surface so that the photopolymer layers would adhere to the glass evenly, producing a layer of uniform thickness.

(b) 1.3-1.4 mLs of the photopolymer solution is then deposited evenly over the area of the glass plate using a syringe.

(c) Using this method the typical material thickness is $100 \pm 10$ microns. Different thicknesses can be obtained by depositing (drop casting) different quantities of material. The thickness and uniformity of these layers can be measured using a micrometer screw gauge.

(d) The plates are then left in the dark for approximately 24 hours until dry. Drying times are dependent on the thickness of the material and the relative humidity.

\section{Behaviors of the Five Photosensitizers in AA/PVA}

4.1. Transmission Spectra. The dyes examined absorb light and act as photoinitiator, of the polymerization process. Figure 2 shows the transmittance of the four Xanthenes dyes, that is. $\mathrm{EB}, \mathrm{EY}, \mathrm{PB}$, and $\mathrm{RB}$ over a range of visible wavelength.

The normalized transmittance $T(t)$ can be expressed as:

$$
T(t)=T_{s f} \exp [-\varepsilon A(t) d]
$$

where $T_{s f}$ is the transmission fraction which corrects for the boundary and scatter losses, $\varepsilon$ is the molar absorption of the

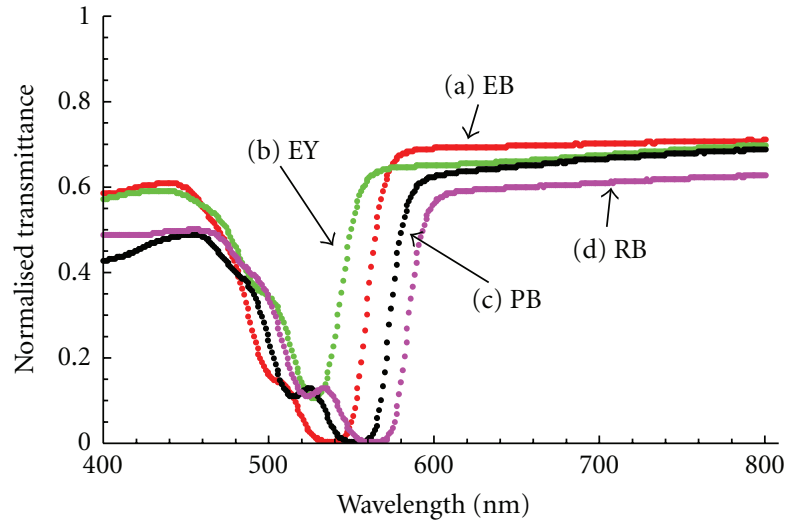

FIgure 2: Spectrum for four different photosensitizers (a) EB (red curve), (b) EY (green curve), (c) PB (black curve), and (d) RB (pink curve) in AA/PVA photopolymer material.

dye, $A(t)$ is the time varying dye concentration, and $d$ is the thickness of the material layer. Taking $t=0$, (14) reduces to:

$$
T_{0}=T_{s f} \exp \left[-\varepsilon A_{0} d\right]
$$

where $T_{0}$ is the transmittance value for one particular wavelength, $\lambda, T_{0}(\lambda)$ is presented in Figure 2, for an initial dye concentration $A_{0}=1.22 \times 10^{-6} \mathrm{~mol} / \mathrm{cm}^{3}$. We define $T_{s f}$ to be the maximum transmittance value. Using this maximum value the molar absorption, $\varepsilon$, for a wavelength of $\lambda=532 \mathrm{~nm}$, that is, the exposing wavelength in all the following experiments, can be estimated.

We also note that the transmittance at a wavelength of $633 \mathrm{~nm}$, for all the dyes is over 59\%, taking into account the increased scatter as a result of the crystallisation of the monomer with the time for this case and the thicker thickness of the layer, this means that $633 \mathrm{~nm}$ laser light can be used as the probe beam during recording. The resulting parameter values estimated are listed in Tables 2 and 3.

4.2. Transmission. In this subsection, we begin by examining the transmission process of the material. In all cases intensities of $10 \mathrm{~mW} / \mathrm{cm}^{2}$ (for $\mathrm{EB}, \mathrm{EY}, \mathrm{PB}$, and $\mathrm{RB}$ ), and $4.03 \mathrm{~mW} / \mathrm{cm}^{2}$ (for $\mathrm{MB}$ ) are used to illuminate the layers. First, we need to examine the effects of adding different dyes to the standard AA/PVA material using simple experiments. In all cases the set-ups involve: uniform plane waves of wavelength $532 \mathrm{~nm}$ (for $\mathrm{EB}, \mathrm{EY}, \mathrm{PB}$, and $\mathrm{RB}$ ) or of $633 \mathrm{~nm}$ (for $\mathrm{MB})$, pass normally through the material and the transmitted intensity is measured. The area of illumination is $0.25 \mathrm{~cm}^{2}$. Normalised transmittance curve for the same dye concentrations for $\mathrm{EB}, \mathrm{EY}, \mathrm{PB}$, and $\mathrm{RB}$, and a higher dye concentration for $\mathrm{MB}$, are given in Figure 3. Using the model proposed in Section 2 dye parameter values are estimated by fitting these curves. Table 4 lists the values of molar absorptivity, $\varepsilon$, and the quantum efficiency of the reactions in which the ground state dye is converted into the singlet ${ }^{1} D^{*}$ and triplet ${ }^{3} D^{*}$ dye states with the quantum efficiencies $\varphi_{S}$ and $\varphi_{T}$ respectively, as estimate by fitting the transmittance curves. We note that the transmittance curves all decrease once exposure ends. This 
TABLE 2: Key parameters for four different Xanthene photosensitizers at a wavelength of $532 \mathrm{~nm}$.

\begin{tabular}{cccccc}
\hline & Type of photosensitizer & Transmittance $(\%)$ & $T_{s f}(\%)$ & $d\left(10^{-4}\right)(\mathrm{cm})$ & $\varepsilon\left(10^{8}\right)\left(\mathrm{cm}^{2} / \mathrm{mol}^{2}\right)$ \\
\hline a & Erythrosine B & 0.6209 & 70.94360 & 200 & 1.942 \\
$\mathrm{~b}$ & Eosin Y & 12.1757 & 69.82245 & 200 & 0.716 \\
$\mathrm{c}$ & Phloxine B & 9.4548 & 68.90019 & 200 & 0.814 \\
$\mathrm{~d}$ & Rose Bengal & 12.8009 & 62.77100 & 200 & 0.652 \\
\hline
\end{tabular}

TABle 3: Transmittance values for four different Xanthene photosensitizers at $633 \mathrm{~nm}$.

\begin{tabular}{ccc}
\hline & Type of photosensitizer & Transmittance $(\%)$ \\
\hline a & Erythrosine B (EB) & 69.4110 \\
b & Eosin Y (EY) & 65.6761 \\
c & Phloxine B (PB) & 64.1600 \\
d & Rose Bengal (RB) & 59.4887 \\
\hline
\end{tabular}

takes place due to the recovery process of the dye once the illuminating light is switched off [18]. $t_{\text {expa }}=100 \mathrm{~s}, t_{\text {expb }}=$ $150 \mathrm{~s}, t_{\text {expc }}=600 \mathrm{~s}, t_{\text {expd }}=200 \mathrm{~s}$, and $t_{\text {expe }}=50 \mathrm{~s}$ are the times when illumination ends for: (a) EB, (b) EY, (c) PB, (d) RB, and (e) $M B$, respectively. It is worth noting that the values of molar absorptivity, $\varepsilon$, estimated here agree with those presented in Section 4.1. The value in Section 4.1 is calculated directly by measuring the spectrum and in Section 4.2 the numbers are estimated by fitting the experimental data. However given to the material and experimental environment difference, that is, temperature and humidity, the value could be slightly different. However we can see the agreement is still surprisingly good, and demonstrates that the absorptivity value extracted using our model is reasonable.

4.3. Recovery. In this section we estimate the rate, $k_{r}$, at which the photosensitizer recovers back to its initial ground state after it has been excited. In order to determine a value for $k_{r}$, a set of experiments is performed, which enables the photosensitizer concentration to be measured at any time, $t$, after a given exposure time, $t_{\exp }$. To do so we use the results presented in Figure 4.

Using the same setup describe in Section 4.2, the recovery process is examined experimentally. The material is illuminated for $t_{\exp }$, after $t_{\exp }$ the laser is switched off for time $t_{\text {off }}$. After $t_{\text {off }}$ [19], the material is again illuminated and the transmittance immediately measured. This measurements repeat several times for different $t_{\text {off }}$ values, so that we obtain the normalised transmission post-exposure, $T\left(t>t_{\exp }\right)$, which is related to the dye concentration post-exposure. As a result, by fitting the experimental data the recovery rate, $k_{r}$, can be estimated.

Based on the best fits achieved to the experimental results, using the model described in Section 2, Table 5 shows the estimated values of the recovery rate, $k_{r}$. The mean squared error (MSE) values achieved for the fitting procedure are also presented in the table.

4.4. Bleaching. In this section the estimation of the rate constant of photobleaching of the photosensitizer, $k_{b}$, the rate

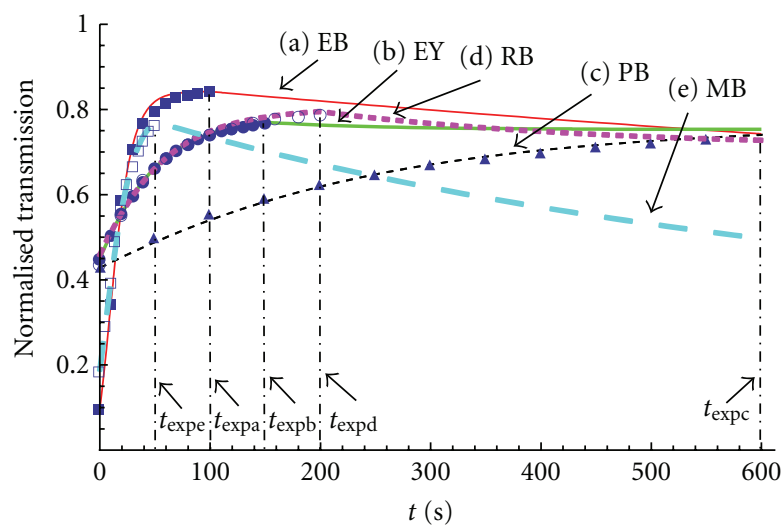

$\begin{array}{ll}\square \text { (a) EB } & \circ \text { (d) RB } \\ \text { (b) EY } & \square \text { (e) MB } \\ \Delta \text { (c) PB } & \end{array}$

FIGURE 3: The normalised transmission characteristics of five different photosensitizers (a) EB (thin red curve and filled square) (b) EY (thick green curve and filled circle) (c) PB (thin short black dashed curve and filled triangle) (d) RB (thick pink short dashed curve and empty circle) (e) MB (thick blue long dashed curve and empty square) in AA/PVA photopolymer material. Both the experimental data points and theoretical fits for exposure intensities of $10 \mathrm{~mW} / \mathrm{cm}^{2}$ for (a), (b), (c), (d), and $4.03 \mathrm{~mW} / \mathrm{cm}^{2}$ for (e) are shown.

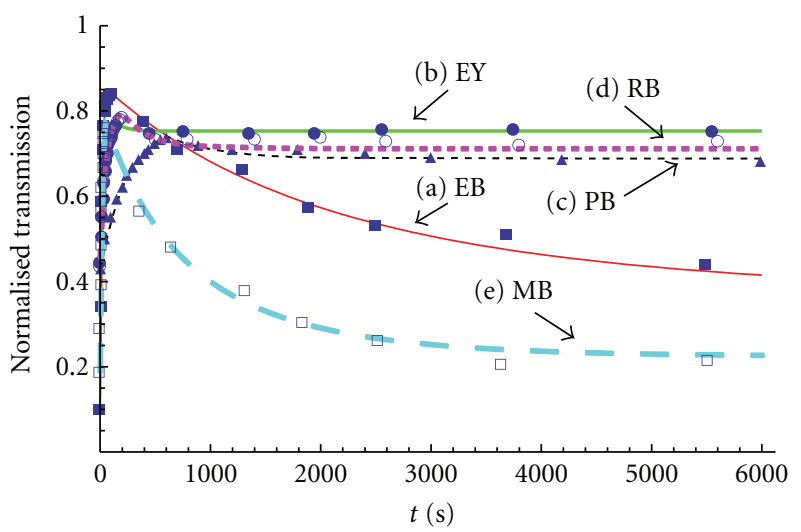

FIGURE 4: The recovery process for five different photosensitizers (a) EB (thin red curve and filled square) (b) EY (thick green curve and filled circle) (c) PB (thin black short dashed curve and filled triangle) (d) RB (thick pink short dashed curve and empty circle) (e) MB (thick blue long dashed curve and empty square) in AA/PVA photopolymer material. Both the experimental data points and theoretical fits for the exposure intensity of $10 \mathrm{~mW} / \mathrm{cm}^{2}$ for (a), (b), (c), (d), and $4.03 \mathrm{~mW} / \mathrm{cm}^{2}$ for (e) are shown. 
TABLE 4: The values of dye absorption-related parameters extracted by fitting the experimental data in Figure 3 using the model in Section 2.

\begin{tabular}{cccccc}
\hline & Type of photosensitizer & $\lambda(\mathrm{nm})$ & $\varepsilon\left(10^{8}\right)\left(\mathrm{cm}^{2} / \mathrm{mol}\right)$ & $\varphi_{S}\left(10^{-3}\right)(\mathrm{mol} /$ Einstein $)$ & $\varphi_{T}\left(10^{-3}\right)(\mathrm{mol} /$ Einstein $)$ \\
\hline a & Erythrosine B & 532 & 1.720 & 6.00 & 9.10 \\
$\mathrm{~b}$ & Eosin Y & 532 & 0.474 & 3.60 & 30.00 \\
$\mathrm{c}$ & Phloxine B & 532 & 0.525 & 1.53 & 4.30 \\
$\mathrm{~d}$ & Rose Bengal & 532 & 0.470 & 8.40 & 19.00 \\
$\mathrm{e}$ & Methylene Blue & 633 & 0.824 & 57.00 & 7.30 \\
\hline
\end{tabular}

TABle 5: The parameter values associated with dye recovery extracted from fitting the experimental results in Figure 4 using model in Section 2 .

\begin{tabular}{ccccc}
\hline & $\lambda(\mathrm{nm})$ & Type of photosensitizers & $k_{r}\left(10^{-3}\right)\left(\mathrm{s}^{-1}\right)$ & MSE \\
\hline $\mathrm{a}$ & 532 & Erythrosine B & 0.33 & 0.01021400 \\
$\mathrm{~b}$ & 532 & Eosin Y & 10.00 & 0.00182489 \\
$\mathrm{c}$ & 532 & Phloxine B & 2.20 & 0.00644423 \\
$\mathrm{~d}$ & 532 & Rose Bengal & 3.90 & 0.01056260 \\
$\mathrm{e}$ & 633 & Methylene Blue & 0.80 & 0.00838055 \\
\hline
\end{tabular}

constant of electron donation (with which $E D$ becomes a free radical, $\left.R^{\bullet}\right), k_{d}$, and the rate constants of inhibition of the photosensitiser, $k_{z}$, dye ${ }^{*}$, are discussed.

The setup for this experiment is the same as that used in Sections 4.2 and 4.3. The material is illuminated for $t_{\exp }$, after $t_{\text {exp }}$ the illumination is switched off for $t_{\mathrm{OFF}}$. The material is left unilluminated for a long time, $t_{\mathrm{OFF}} \rightarrow \infty$ in order to allow the dye to fully recover. In practise, we assume that the dye is fully recovered after $t_{\mathrm{OFF}}=12$ hours. After $t_{\mathrm{OFF}}=12$ hours the illumination is once again switched on and the transmittance measured. This procedure is repeated several times with different $t_{\exp }$ values for all the dyes.

The amount of photosensitizer concentration bleached by an exposure of duration, $t_{\text {exp }}$, can be estimated by taking the differences between the initial concentration $A_{0}$ and $A\left(t_{\mathrm{OFF}} \rightarrow \infty\right)$,

$$
A_{b}=A_{0}-A\left(t_{\mathrm{OFF}} \longrightarrow \infty\right),
$$

where $A_{0}$ is the initial photosensitizer concentration and $A\left(t_{\mathrm{OFF}} \rightarrow \infty\right)$ is the final value. A typical set of experimental results are shown in Figure 5, and the resulting parameter values estimated are listed in Table 6.

4.5. Summary. In this section, the photoabsorptive behaviours of five photosensitizers, in almost equivalent PVA/AA layers, are examined using the model derived in Section 2. For each dye, the recovery and bleaching parameters are estimated by fitting reproducible experimental transmittance data.

It can be noted from the quality of the fitting results that the RB is not as well modelled as the other dyes, in that at a certain time $t$, the sum of the concentration of the recovered dye and the bleached dye is less than the original dye concentration value $A_{0}$. Furthermore the quality of the fit is poorer as indicated by the fact that the MSE value is relatively higher. One possibility is that this could be a result of our neglect of the recovery of the triplet excited state dye, ${ }^{3}$ Dye ${ }^{*}$ back into either the ground state, Dye,or the singlet state,

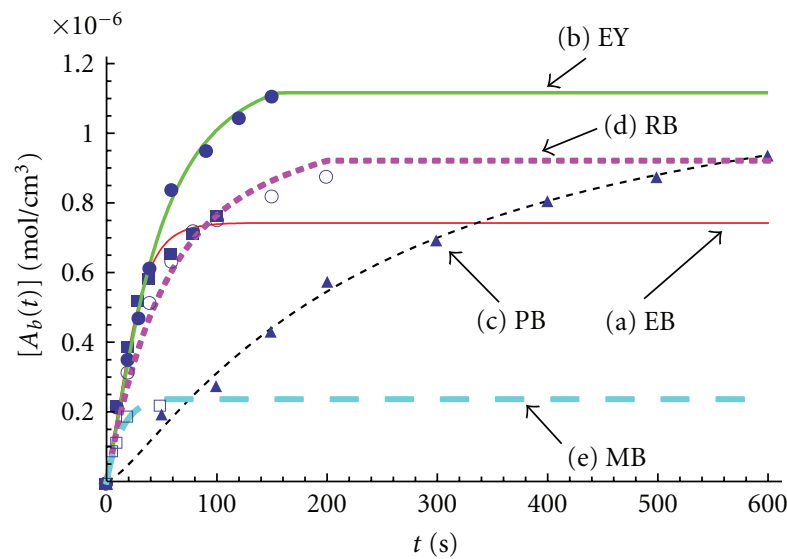

FIGURE 5: The bleaching process for five different photosensitizers (a) EB (thin red curve and filled square), (b) EY (thick green curve and filled circle), (c) PB (thin black short dashed curve and filled triangle), (d) RB (thick pink short dashed curve and empty circle), (e) MB (thick blue long dashed curve and empty square) in AA/PVA photopolymer material. All the experimental data points and theoretical fits for the exposure intensity of $10 \mathrm{~mW} / \mathrm{cm}^{2}$ for (a), (b), (c), and (d), and $4.03 \mathrm{~mW} / \mathrm{cm}^{2}$ for (e) are shown. In all the cases the illuminated area was $0.25 \mathrm{~cm}^{2}$.

${ }^{1}$ Dye ${ }^{*}$. However, following some analyses we note that even if such recovery mechanisms are included into the model the quality of the fit does not improve significantly. There is a connection between the recovery and the bleaching process. Using the same experiment data, as is presented in Figures 6 and 7 it is found that when we get the best fitting for the transmission and recovery of $\mathrm{RB}$, the theoretical predicted values are higher than the experimental values for bleaching, especially for the longest values of time. Table 7 lists the parameter values extracted by applying this procedure. In conclusion, based on these observations, we expect that there are other reactions taking place in the $\mathrm{RB}$ system, which are of less significance for the other dyes studied. 
TABLE 6: The values of bleaching-related parameters extracted by fitting experimental date in Figure 5 using the model in Section 2.

\begin{tabular}{|c|c|c|c|c|c|c|}
\hline & Dye & $\lambda(\mathrm{nm})$ & $k_{b}\left(\mathrm{~cm}^{3} / \mathrm{mol} \mathrm{s}\right)$ & $k_{z, \text { dye* }}\left(\mathrm{cm}^{3} / \mathrm{mol} \mathrm{s}\right)$ & $k_{d}\left(10^{3}\right)\left(\mathrm{cm}^{3} / \mathrm{mol} \mathrm{s}\right)$ & $\operatorname{MSE}\left(\times 10^{-8}\right)$ \\
\hline $\mathrm{a}$ & $\mathrm{EB}$ & 532 & $0.23 \times 10^{2}$ & $1.1 \times 10^{8}$ & 4.3 & 2.019090 \\
\hline b & EY & 532 & $0.80 \times 10^{2}$ & $1.5 \times 10^{8}$ & 3.8 & 1.840540 \\
\hline c & $\mathrm{PB}$ & 532 & $0.20 \times 10^{2}$ & $5 \times 10^{7}$ & 3.9 & 1.387100 \\
\hline d & $\mathrm{RB}$ & 532 & $6.000 \times 10^{3}$ & $9 \times 10^{7}$ & 5.0 & 2.607790 \\
\hline e & MB & 633 & $4.0 \times 10^{7}$ & $1.0 \times 10^{8}$ & 4.3 & 0.981322 \\
\hline
\end{tabular}

TABLE 7: The parameters extracted by fitting experimental date in Figure 7 using the alternate model for RB.

\begin{tabular}{|c|c|c|c|c|}
\hline$\varepsilon\left(10^{8}\right)\left(\mathrm{cm}^{2} / \mathrm{mol}\right)$ & $\varphi_{S}\left(10^{-3}\right)(\mathrm{mol} /$ Einstein $)$ & $\varphi_{T}\left(10^{-3}\right)(\mathrm{mol} /$ Einstein $)$ & $k_{r}\left(10^{-3}\right)\left(\mathrm{s}^{-1}\right)$ & MSE for recovery \\
\hline 0.510 & 8.40 & 17.90 & 9.10 & 0.00251136 \\
\hline$k_{b}\left(\mathrm{~cm}^{3} / \mathrm{mol} \mathrm{s}\right)$ & $k_{z, \text { dye* }}\left(\mathrm{cm}^{3} / \mathrm{mol} \mathrm{s}\right)$ & $k_{d}\left(10^{3}\right)\left(\mathrm{cm}^{3} / \mathrm{mol} \mathrm{s}\right)$ & \multicolumn{2}{|c|}{ MSE for bleaching $\left(\times 10^{-8}\right)$} \\
\hline $6.000 \times 10^{3}$ & $9 \times 10^{7}$ & 5.0 & \multicolumn{2}{|c|}{3.988450} \\
\hline
\end{tabular}

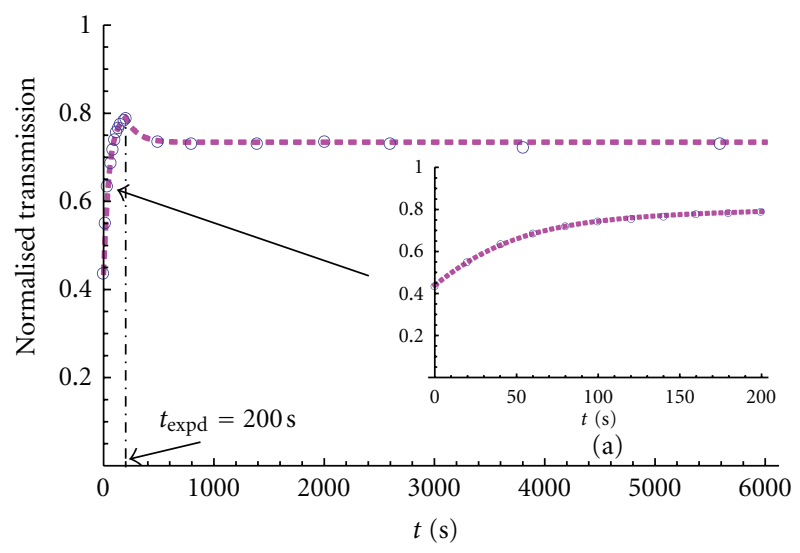

(b)

Figure 6: (a) The normalised transmission characteristics RB. (b) The recovery process for RB in AA/PVA photopolymer material. Both the experimental data points (circles) and theoretical fit (dashed line) for an exposure intensity of $10 \mathrm{~mW} / \mathrm{cm}^{2}$ are shown for the new fitting.

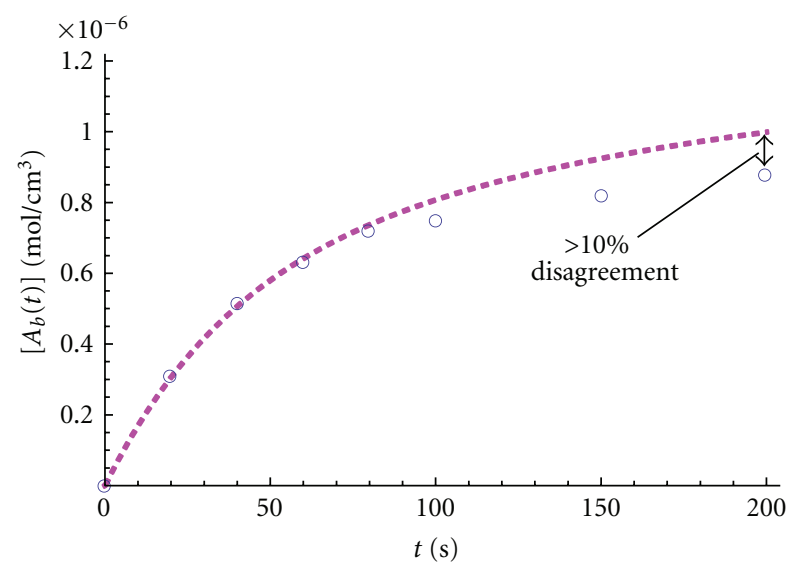

FIgURE 7: The bleaching process for RB in AA/PVA photopolymer material. Both the experimental data points and theoretical fits for the exposure intensity of $10 \mathrm{~mW} / \mathrm{cm}^{2}$ are shown with the new fitting. In all the cases, the illuminated area was $0.25 \mathrm{~cm}^{2}$.

\section{Simulation Model Predictions}

Based on the theoretical analysis and experimental work presented in Sections 2 to 4, the temporal evolution of the primary radical concentration, $E D^{\bullet}$ ( or $R^{\bullet}$ ), can be predicted. It is found that the behaviours of the five dyes are significantly different.

From Section 2, we know that the primary radical concentration, $E D^{\bullet}$, is produced by the reaction between ${ }^{3} \mathrm{Dye}^{*}$ and $E D$, at the rate constant of electron donation, $k_{d}$, see (1d). Simultaneously, $E D^{\bullet}$ is also being removed as it initiates photopolymer chains, see (1f), as it reacts with $M_{n}^{\cdot}$, see (4), and is scavenged by the dissolved inhibitor, see (5a). Applying the full model of the photoinitiation process, using the parameter values estimated above, the temporal evolution of the concentration of the primary radicals, $E D^{\circ}$, for (a) EB, (b) EY, (c) PB, (d) RB, and (e) MB, can be predicted using (8).

The result is presented in Figure 8. As can be seen in the initial stages of the exposure, the generation of $E D^{\bullet}$ is significantly inhibited by the original dissolved oxygen, see (5a). Following the resulting initial inhibition period, the generation of $E D^{\bullet}$ progressively increases towards a maximum value. However, after this maximum the concentration value decreases gradually. This is due to the photoinitiation process described by (1f), and the reaction with $M_{n}^{\bullet}$ during the termination process described in (4).

From Figure 8 we can see that for the four Xanthene dyes examined, EB reaches the $E D^{\bullet}$ peak concentration value in a very short time and the $E D^{\circ}$ peak concentration is also highest, which means that the inhibitor has less effect for the EB case. However the inhibition effect can be more accurately measured using lower exposure intensity, and this is a topic for future work. In addition, for the corresponding MB case, the general behaviour of the $E D^{\circ}$ concentration is similar to that of the Xanthene dyes.

For $\mathrm{PB}$ and $\mathrm{MB}$, since fewer ${ }^{3} \mathrm{Dye}{ }^{*}$ molecules are generated, fewer are available to react with the electron donor, that is, $\varphi_{\mathrm{T}}(\mathrm{PB})$ and $\varphi_{\mathrm{T}}(\mathrm{MB})$ are smaller, thus less $E D^{\circ}$ is produced. Therefore: (1) the inhibitor scavenging effect takes less time to end; (2) at the same corresponding time in Figure 8, the amount of dihydro dye formed is reduced, that 


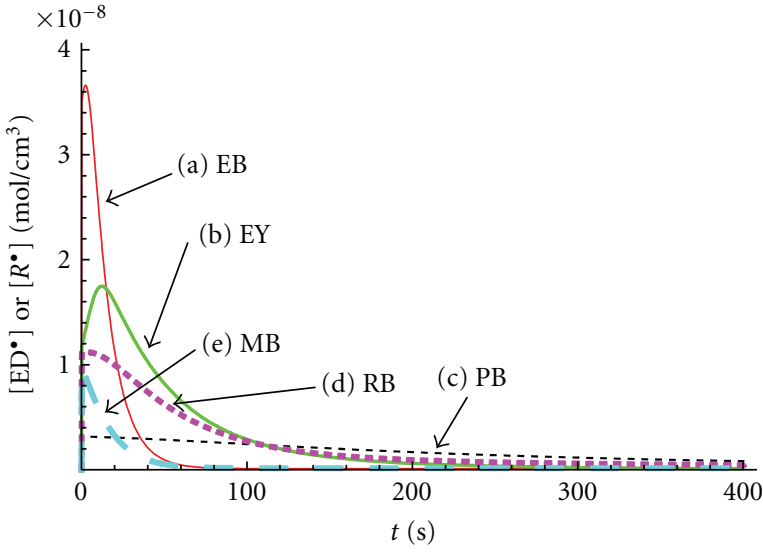

FIgURE 8: The predicted concentration of $E D^{\bullet}$, or $R^{\bullet}$, from (8) as a function of time for: (a) EB (thin red curve), (b) EY (thick green curve), (c) PB (thin black short dashed curve), (d) RB (thick pink short dashed curve), (e) MB (thick blue long dashed curve), in AA/PVA photopolymer material layer.

is, there is less bleaching of the $\mathrm{PB}$ and $\mathrm{MB}$ dyes for the same exposure time, see Figure 5.

Furthermore, comparing the results for these five types of photosensitizer, it is clear that the generation of primary radicals can be affected by the different reactivities between the $E D$ molecules and the various excited dye molecules. This is important in the photoinitiation process, especially if one wishes to optimize the interaction between the electron donor and the photosensitizer. Therefore, and in agreement with previous results in the literature, in order to optimize a free-radical polymerization process $[1,16,22,25,26,32,33]$, it is clear that a detailed model of dye kinetics, that is, photon absorption, photosensitizer recovery and bleaching is necessary. Once again we note that the behaviour of RB dye is different from that of the others, and once again this may be due to the presence of some other process.

\section{Conclusion and Analysis}

One of the aims of this paper has been the development of a new dye model which is both simple enough to use easily but also of practical value and sufficiently flexible to be used for different photosensitisers. In this paper, key dye parameter values for five photosensitizers, (i.e., molar absorptivity, $\varepsilon$, quantum efficiency of the reaction, $\varphi_{S}$ and $\varphi_{T}$, recovery rate, $k_{r}$, and bleaching rate, $k_{b}$ ), have been extracted by fitting the experiment data using our new simplified dye model. This is achieved by measuring the transmission of the material layer, for certain exposure intensities. The recovery behaviours of the dyes are thus experimentally compared under identical conditions. Following this, the bleaching processes [19] were examined using the parameter values already estimated. In this way a full theoretical treatment of the photoinitiation processes was presented. The results are very useful, that is, $\mathrm{PB}$ should be used to record gratings with low energetic exposure, in that the inhibition has a less effect on it.

While significant progress has been made in the work presented here, much remains to be done. It appears that some other reactions are taking placing during and after-exposure in the RB system case, indicating that a more accurate model is needed. The resulting accurate description of the photoinitiation processes must then be incorporated into the full nonlocal photopolymerization driven diffusion (NPDD) model, allowing a more complete modelling of free radical photopolymerization to take place. Such a model can then be applied to the study of photopolymer use for such application as holographic data storage and 3D hybrid optoelectronic circuit fabrication.

A new dye model, which includes the effect of photosensitizer recovery and bleaching has been developed and compared to experimental results. The photoabsorptive behaviours of five types of photosensitizer: Erythrosine B (EB); Eosin Y (EY); Phloxine B (PB); and Rose Bengal (RB), of the same molarity in equivalent PVA/AA layers, and Methylene Blue (MB) of higher molarity in PVA/AA layers, are experimentally examined. We modify the previous dye model by introducing $k_{a S}$ and $k_{a T}$ into the model, which are the rate constant of photon absorption from ground state dye to singlet state and triplet state respectively. A description of how to incorporate this dye model into a full NPDD material model, in order to simulate photopolymer material behaviour, has been presented and discussed.

Future work must include characterising the spatial frequency response of the AA/PVA material. There is a large difference between the molecular weights between five dyes. It is reasonable to assume that, barring an unusual molecular structure, the smaller the molecule, the quicker it will diffuse through a given polymer matrix [34]. It is expected that the faster the rate of diffusion, the more total polymerisation that can take place in the exposed areas. This should lead to a larger saturation value of refractive index modulation and a stronger material response. In summary the effect of the five different polarities, shapes and size of the Xanthene and Thiazine dyes on the spatial frequency response of the AA/PVA material must be quantitatively examined $[35,36]$. Since the existence and diffusion of oxygen is non-ignorable in low intensity exposure cases, the inhibition process will be studied by using low exposure intensity in the future.

\section{Acknowledgments}

The authors would like to acknowledge the funding support of the EU ERUSMAS Mundus fund. They would also like to acknowledge the support of Enterprise Ireland and Science Foundation Ireland under the national development fund and the Irish Research Council for Science, Engineering and Technology (IRCSET) through the EMPOWER postgraduate and postdoctoral fellowship schemes and the Ministerio de Economía y Competitividad of Spain under Project FIS201129803-C02-01 as well.

\section{References}

[1] M. R. Gleeson, D. Sabol, S. Liu, C. E. Close, J. V. Kelly, and J. T. Sheridan, "Improvement of the spatial frequency response of photopolymer materials by modifying polymer chain length," Journal of the Optical Society of America B, vol. 25, no. 3, pp. 396-406, 2008. 
[2] C. H. Bamford, A. D. Jenkins, and R. Johnston, "Termination by primary radicals in vinyl polymerization," Transactions of the Faraday Society, vol. 55, pp. 1451-1460, 1959.

[3] G. Manivannan and R. A. Lessard, "Trends in holographic recording materials," Trends in Polymer Science, vol. 2, pp. 282290, 1994.

[4] A. Márquez, C. Neipp, A. Beléndez, S. Gallego, M. Ortuño, and I. Pascual, "Edge-enhanced imaging with polyvinyl alcohol/ acrylamide photopolymer gratings," Optics Letters, vol. 28, no. 17, pp. 1510-1512, 2003.

[5] S. Harbour, J. V. Kelly, T. Galstian, and J. T. Sheridan, "Optical birefringence and anisotropic scattering in acrylate based holographic polymer dispersed liquid crystals," Optics Communications, vol. 278, no. 1, pp. 28-33, 2007.

[6] S. M. Schultz, E. N. Glytsis, and T. K. Gaylord, "Design, fabrication, and performance of preferential-order volume grating waveguide couplers," Applied Optics, vol. 39, no. 8, pp. 12231232,2000

[7] A. Sato, M. Scepanovic, and R. K. Kostuk, "Holographic edgeilluminated polymer Bragg gratings for dense wavelength division optical filters at $1550 \mathrm{~nm}$," Applied Optics, vol. 42, no. 5, pp. 778-784, 2003.

[8] M. Straub, L. H. Nguyen, A. Fazlic, and M. Gu, "Complexshaped three-dimensional microstructures and photonic crystals generated in a polysiloxane polymer by two-photon microstereolithography," Optical Materials, vol. 27, no. 3, pp. 359-364, 2004.

[9] A. C. Sullivan, M. W. Grabowski, and R. R. McLeod, "Threedimensional direct-write lithography into photopolymer," Applied Optics, vol. 46, no. 3, pp. 295-301, 2007.

[10] J. V. Kelly, M. R. Gleeson, C. E. Close, and J. T. Sheridan, "Optimized scheduling for holographic data storage," Journal of Optics A, vol. 10, no. 11, Article ID 115203, 2008.

[11] L. Dhar, A. Hale, H. E. Katz, M. L. Schilling, M. G. Schnoes, and F. C. Schilling, "Recording media that exhibit high dynamic range for digital holographic data storage," Optics Letters, vol. 24, no. 7, pp. 487-489, 1999.

[12] R. R. McLeod, A. J. Daiber, M. E. McDonald et al., "Microholographic multilayer optical disk data storage," Applied Optics, vol. 44, no. 16, pp. 3197-3207, 2005.

[13] F. Bruder and T. Faecke, "Materials in optical data storage," International Journal of Materials Research, vol. 101, pp. 199$215,2010$.

[14] J. Zhang, K. Kasala, A. Rewari, and K. Saravanamuttu, "Selftrapping of spatially and temporally incoherent white light in a photochemical medium," Journal of the American Chemical Society, vol. 128, no. 2, pp. 406-407, 2006.

[15] M. R. Gleeson, J. V. Kelly, C. E. Close, F. T. O’Neill, and J. T. Sheridan, "Effects of absorption and inhibition during grating formation in photopolymer materials," Journal of the Optical Society of America B, vol. 23, no. 10, pp. 2079-2088, 2006.

[16] M. R. Gleeson, J. V. Kelly, D. Sabol, C. E. Close, S. Liu, and J. T. Sheridan, "Modeling the photochemical effects present during holographic grating formation in photopolymer materials," Journal of Applied Physics, vol. 102, no. 2, Article ID 023108, 9 pages, 2007.

[17] M. R. Gleeson, S. Liu, S. O’Duill, and J. T. Sheridan, "Examination of the photoinitiation processes in photopolymer materials," Journal of Applied Physics, vol. 104, no. 6, Article ID 064917, 8 pages, 2008.

[18] S. Liu, M. R. Gleeson, D. Sabol, and J. T. Sheridan, "Extended model of the photoinitiation mechanisms in photopolymer materials," Journal of Applied Physics, vol. 106, no. 10, Article ID 104911, 10 pages, 2009.
[19] S. Liu, M. R. Gleeson, J. Guo, and J. T. Sheridan, "Optical characterization of photopolymers materials: theoretical and experimental examination of primary radical generation," Applied Physics B, vol. 100, no. 3, pp. 559-569, 2010.

[20] J. R. Lawrence, F. T. O’Neill, and J. T. Sheridan, "Photopolymer holographic recording material," Optik, vol. 112, no. 10, pp. 449-463, 2001.

[21] A. Fimia, N. López, F. Mateos, R. Sastre, J. Pineda, and F. Amat-Guerri, "New photopolymer used as a holographic recording material," Applied Optics, vol. 32, no. 20, pp. 37063707, 1993.

[22] M. D. Goodner and C. N. Bowman, "Modeling primary radical termination and its effects on autoacceleration in photopolymerization kinetics," Macromolecules, vol. 32, no. 20, pp. 6552-6559, 1999.

[23] H. K. Mahabadi, "Effects of chain length dependence of termination rate constant on the kinetics of free-radical polymerization. 1. Evaluation of an analytical expression relating the apparent rate constant of termination to the number-average degree of polymerization," Macromolecules, vol. 18, no. 6, pp. 1319-1324, 1985.

[24] G. Odian, Principles of Polymerization, John Wiley \& Sons, New York, NY, USA, 4th edition, 1991.

[25] M. R. Gleeson and J. T. Sheridan, "Nonlocal photopolymerization kinetics including multiple termination mechanisms and dark reactions. Part I. Modeling," Journal of the Optical Society of America B, vol. 26, no. 9, pp. 1736-1745, 2009.

[26] M. R. Gleeson, S. Liu, R. R. McLeod, and J. T. Sheridan, "Nonlocal photopolymerization kinetics including multiple termination mechanisms and dark reactions. Part II. Experimental validation," Journal of the Optical Society of America B, vol. 26, no. 9, pp. 1746-1754, 2009.

[27] M. R. Gleeson, S. Liu, J. Guo, and J. T. Sheridan, "Non-local photo-polymerization kinetics including multiple termination mechanisms and dark reactions: part III. Primary radical generation and inhibition," Journal of the Optical Society of America Bp, vol. 27, no. 9, pp. 1804-1812, 2010.

[28] B. B. Bhowmik and P. Ganguly, "Photophysics of xanthene dyes in surfactant solution," Spectrochimica Acta-Part A, vol. 61, no. 9, pp. 1997-2003, 2005.

[29] M. Izadifard, C. H. Langford, and G. Achari, "Photocatalytic dechlorination of PCB 138 using leuco-methylene blue and visible light; reaction conditions and mechanisms," Journal of Hazardous Materials, vol. 181, no. 1-3, pp. 393-398, 2010.

[30] A. K. O'Brien and C. N. Bowman, "Modeling the effect of oxygen on photopolymerization kinetics," Macromolecular Theory and Simulations, vol. 15, no. 2, pp. 176-182, 2006.

[31] J. T. Sheridan and J. R. Lawrence, "Nonlocal-response diffusion model of holographic recording in photopolymer," Journal of the Optical Society of America A, vol. 17, no. 6, pp. 1108-1114, 2000.

[32] M. R. Gleeson, S. Liu, and J. T. Sheridan, "The production of primary radicals in photopolymers during holographic exposure," Optik, vol. 121, no. 24, pp. 2273-2275, 2010.

[33] M. R. Gleeson and J. T. Sheridan, "A review of the modelling of free-radical photopolymerization in the formation of holographic gratings," Journal of Optics A, vol. 11, no. 2, Article ID 024008, 2009.

[34] J. Guo, S. Liu, M. R. Gleeson, and J. T. Sheridan, "Study of photosensitizer diffusion in a photopolymer material for holographic applications," Optical Engineering, vol. 50, no. 1, Article ID 015801, 5 pages, 2011. 
[35] C. E. Close, M. R. Gleeson, and J. T. Sheridan, "Monomer diffusion rates in photopolymer material. Part I. Low spatial frequency holographic gratings," Journal of the Optical Society of America B, vol. 28, no. 4, pp. 658-666, 2011.

[36] C. E. Close, M. R. Gleeson, D. A. Mooney, and J. T. Sheridan, "Monomer diffusion rates in photopolymer material. Part II. High-frequency gratings and bulk diffusion," Journal of the Optical Society of America B, vol. 28, no. 4, pp. 842-850, 2011. 

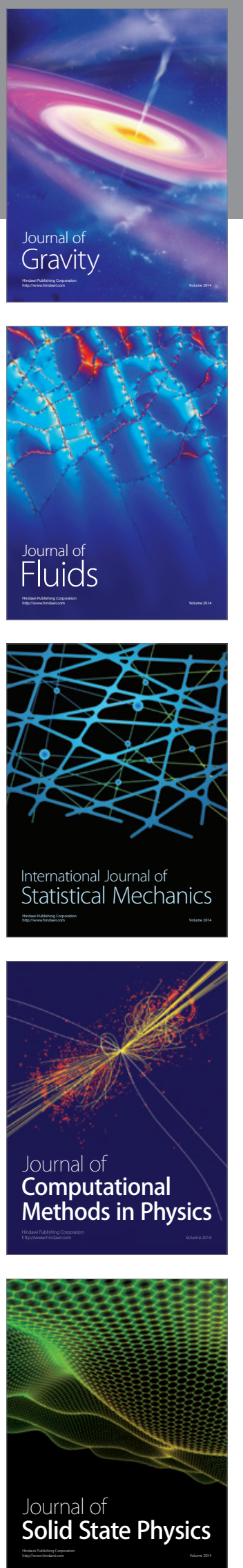

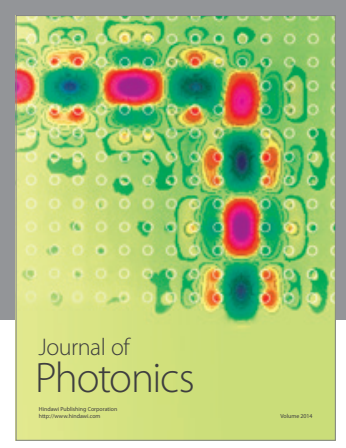

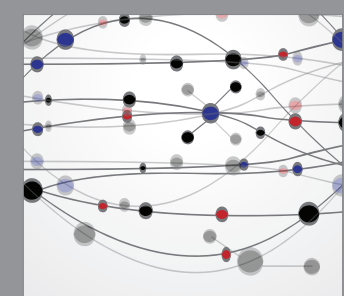

The Scientific World Journal
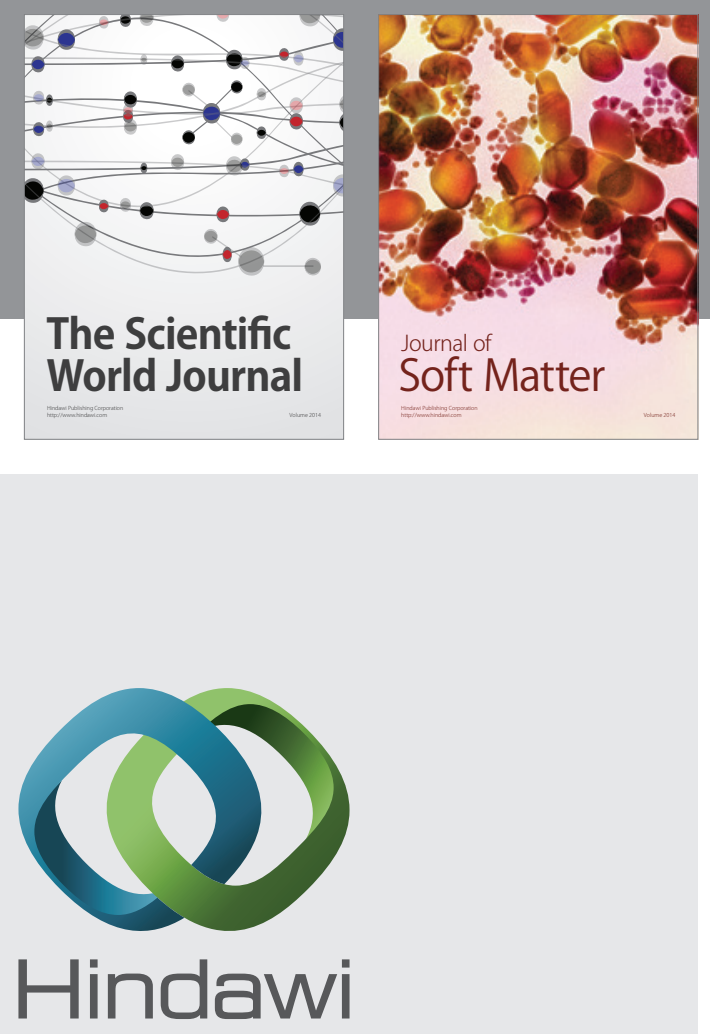

Submit your manuscripts at

http://www.hindawi.com
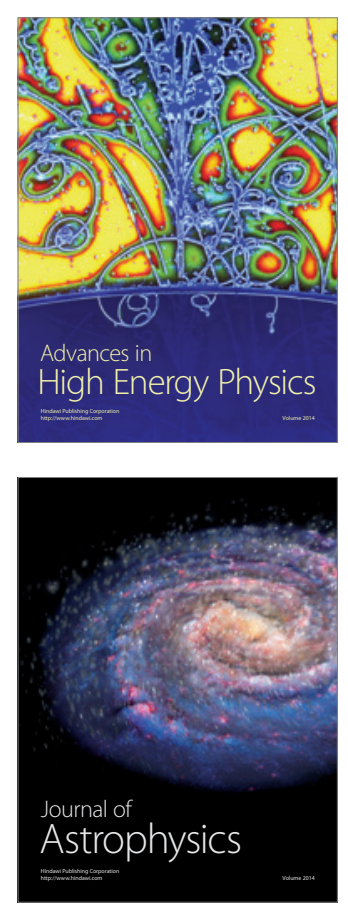
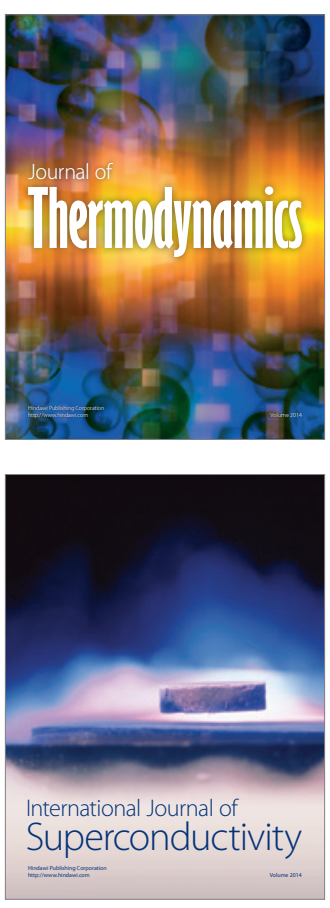
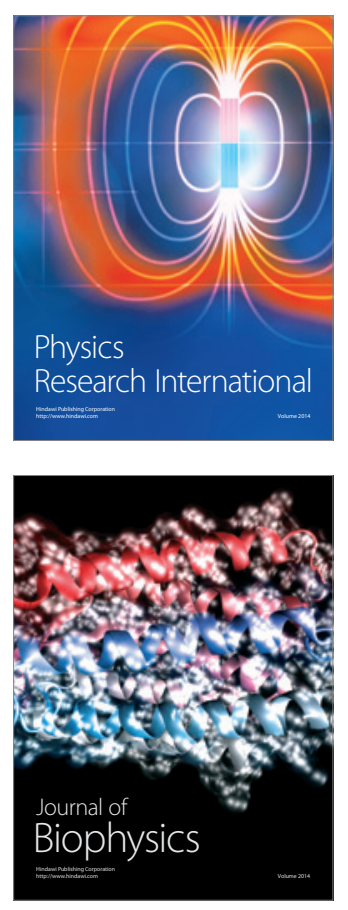
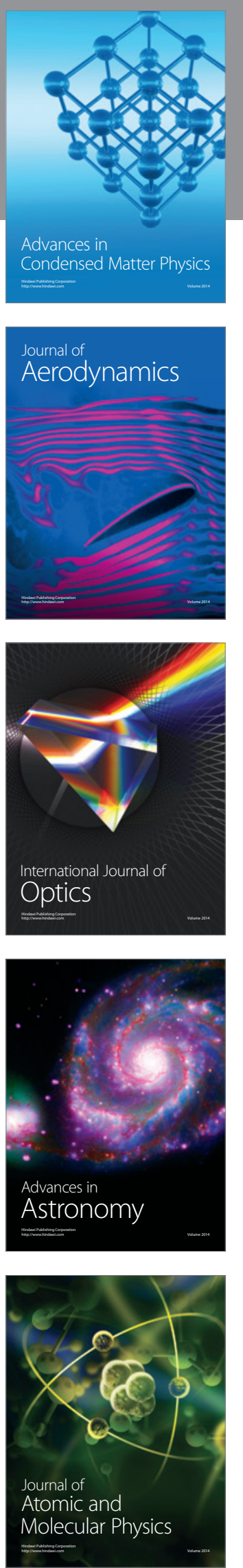